\title{
Editorial : progress in the science and technology of nuclear reactors using molten salts
}

\author{
Jan Leen Kloosterman ${ }^{1,}$, Elsa Merle ${ }^{2}$, and Jean C. Ragusa ${ }^{3}$ \\ 1 TU Delft, The Netherlands \\ ${ }^{2}$ CNRS, France \\ 3 Texas A\&M University, USA
}

Received: 12 December 2019 / Accepted: 12 December 2019

Dear Reader,

Molten salt reactor research and development activities are on a clear, upward trend across the globe, including in China, Europe, the Russian Federation, and the USA. Current both the Fluoride-salt cooled High temperature Reactors (FHR), mostly nuclear reactor designs adapting TRISO fuel and a fluoride salt coolant, and the Molten Salt Reactors (MSR) with actinides and fission products dissolved in the fuel salt. The designs of both reactor classes have several research themes in common, such as structural materials, thermo-physical properties of liquid salts, the interaction of salts with structural materials, thermo-dynamics of salt flow, modelling and simulation, salt freezing phenomena, salt chemistry, tritium production, conversion of high temperature heat to electricity and process heat, and many others.

Besides these common research themes, each reactor type has its own specific research topics and particular reactor concepts. In the field of MSR, for example, a large variety exists with neutron spectra ranging from thermal to fast, fluoride or chloride fuel salts, single flow or multiple flow designs, and designs with a circulating liquid fuel salt or a static fuel salt in rods. We have seen designs aiming at breeding new material from thorium or uranium, and designs with the goal to burn plutonium and other actinides from the existing light-water reactor fleet. Beyond the rationale for the different concepts, researchers in the field share a common goal: we are all motivated to make nuclear energy safer and more sustainable so that it can play a vital role as a stable and safe energy supply and contribute effectively mitigating global warming.

The progress in the EU SAMOFAR project and the US NUSTEM project has inspired us to assemble this special issue of EPJ-N with an overview of research in various fields connected to FHR and MSR. We are grateful to the publisher for this opportunity and we hope you will enjoy reading about the latest results in this exciting field.

\footnotetext{
* e-mail: J.L.Kloosterman@tudelft.nl
} 